\title{
Reflection of light from a disordered medium backed by a phase-conjugating mirror
}

\author{
J C J Paasschens, ${ }^{12}$ M J M de Jong, ${ }^{1}$ P W Brouwer, ${ }^{2}$ and C W J Beenakker ${ }^{2}$ \\ ${ }^{1}$ Philips Research Laboratorles, 5656 AA Eindhoven, The Netherlands \\ ${ }^{2}$ Instituut-Lorentz, Letden Untversitv, PO Box 9506, 2300 RA Leiden, The Netherlands
}

(Recerved 12 May 1997)

\begin{abstract}
This is a theoretical study of the interplay of optical phase conjugation and multiple scattering We calculate the intensity of light reflected by a phase-conjugating mirror when it is placed behind a disordered medium We compare the results of a fully phase-coherent theory with those from the theory of radiative transfer Both methods are equivalent if the dwell time $\tau_{\text {dwell }}$ of a photon in the disordered medium is much larger than the inverse of the frequency shift $2 \Delta \omega$ acquited at the phase conjugating mirror When $\tau_{\mathrm{dwell}} \Delta \omega \leq 1$, in contrast, phase coherence drastically affects the reflected intensity In particular, a minimum in the dependence of the reflectance on the disorder strength disappeats when $\Delta \omega$ is reduced below $1 / \tau_{\text {dwell }}$ The analogies and differences with Andreev reflection of electrons at the interface between a normal metal and a superconductor are discussed [S1050 2947(97)06611-0]
\end{abstract}

PACS number(s) $4265 \mathrm{Hw}, 4225 \mathrm{Bs}, 4268 \mathrm{Ay}, 7820 \mathrm{Cl}$

\section{INTRODUCTION}

Phase conjugation is the reversal of the sign of the phase of a wave function A phase-conjugated wave retraces the path of the original wave, thereby canceling all accumulated phase shifts Phase conjugation was first discovered for electronıc waves [1], and later for optical waves [2,3] For electrons, phase conjugation takes place at the interface between a normal metal and a superconductor An electron at energy $E$ above the Fermi energy $E_{\Gamma}$ is reflected at the angle of incidence (retroreflected) as a hole at energy $E$ below $E_{F}$, a process known as Andreev reflection [4] A phaseconjugating mirror for light consists of a cell containing a liquid or crystal with a large nonlinear susceptibility, pumped by two counterpropagating beams at frequency $\omega_{0}$ A wave incident at frequency $\omega_{0}+\Delta \omega$ is then retroreflected at frequency $\omega_{0}-\Delta \omega$, a process known as tour-wave mixing [5-7]

The interplay of multiple scattering by disorder and phase conjugation has been studied extensively in the electronic case, both experimentally and theoretically (See Ref [8] for a review ) In the optical case the emphasis has been on weakly disordered media, which do not strongly scatter the waves [9] Complete wave-front reconstruction is possible only if the distorted wave front remains approximately planar, since perfect time reversal upon reflection holds only in a narrow range of angles of incidence for realistic systems (For the hypothetical case of perfect time reversal at all angles, see Ref [10] ) McMichael, Ewbank, and Vachss [11] measured the intensity of the reconstructed wave front for a strongly inhomogeneous medium (small transmission probability $T_{0}$ ), and found that it was proportional to $T_{0}^{2}-1 \mathrm{n}$ agreement with the theoretical prediction of $\mathrm{Gu}$ and Yeh [12] If $T_{0} \ll 1$, the intensity of the reconstructed wave is much smaller than the total ieflected intensity The total reflected intensity was not studied previously, perhaps because it was beheved that the diffusive illumination resulting from a strongly inhomogeneous medium would render the effect of phase conjugation insignificant In this paper we show that a strongly disordered medium backed by a phase-conjugatıng mirror has unusual optical properties, different both from the weakly disordered case and from the electionic analog

We distinguish two regimes, depending on the relative magnitude of the frequency shift $2 \Delta \omega$ acquired at the phaseconjugating mirror and the inverse of the dwell time $\tau_{\mathrm{dwell}}$ of a photon in the disordered medium (For a medium of length $L$ and mean free path $l$, with light velocity $c$, one has $\tau_{\text {dwell }} \simeq L^{2} / c l$ ) In the coherent regime, $\Delta \omega \ll 1 / \tau_{\text {dwell }}$, phase conjugation leads to a constructive interference of multiply scattered light in the disordered medium In the incoherent regıme, $\Delta \omega \gg 1 / \tau_{\text {dwell }}$, interference effects are insignificant In both regimes we compute the reflectances $R_{+}$and $R$, defined as the reflected power at frequency $\omega_{0} \pm \Delta \omega$ divided by the incident power at frequency $\omega_{0}+\Delta \omega \mathrm{A}$ distinguishing feature of the two regimes is that (in a certain parameter range) the reflectance $R_{-}$decreases monotonically as a function of $L / l$ in the coherent regime, while in the incoherent regime it first decreases and then increases

The outline of this paper is as follows After having formulated the problem in Sec II, we discuss in Sec III its solution using the Boltzmann equation, Ignoring phase coherence This is the theory of radiative transfer $[13,14]$ A simple result is obtained if we neglect angular correlations between the scattering in the disordered medium and at the phase-conjugatıng mirror We compare this approximation with an exact solution of the Boltzmann equation In Sec IV the phase-coheient problem is addressed, analytically using random-matrix theory, and numerically using the method of 1ecursive Green functions Results of this section were briefly presented in Ref [15] We conclude in Sec V with a comparison with the electronic analog of this problem

\section{FORMULATION OF THE PROBLEM}

We study the system shown in Fig 1 It consists of a disordered medium (length $L$, mean free path $l$ ), backed at one end by a phase-conjugating mirror The other end is illuminated diffusively at frequency $\omega_{+}=\omega_{0}+\Delta \omega$, where $\omega_{0}$ is the pump frequency of the mirror We are interested in 


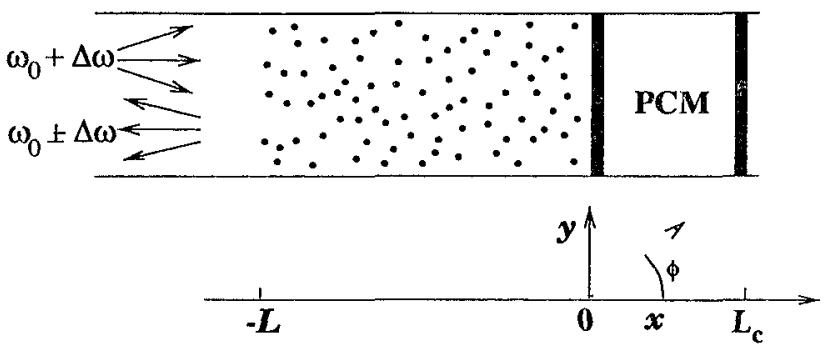

FIG 1 Schematic drawing of the disordered medium backed by a phase conjugating mirror (PCM) Light incident at frequency $\omega_{0}+\Delta \omega$ is reflected at the two frequencies $\omega_{0} \pm \Delta \omega$

the amount of light reflected at frequency $\omega_{+}$and $\omega_{-}=\omega_{0}-\Delta \omega$

To reduce the problem to the scattering of a scalar wave, we choose a two-dimensional geometry The scatterers consist of dielectric rods in the $z$ direction, randomly placed in the $x-y$ plane The electric field points in the $z$ direction and varies in the $x-y$ plane only Two-dimensional scatterers are somewhat artuficial, but can be realized experimentally [16] We believe that our results apply qualitatively to a threedimensional geometry as well, because the iandomization of the polarization by the disorder ienders the vector character of the light insignificant

The $z$ component of the electric field at the frequencies $\omega_{+}$and $\omega_{-}$is given by

$$
E_{ \pm}(x, y, t)=\operatorname{Re} \mathcal{E}_{ \pm}(x, y) \exp \left(-\imath \omega_{ \pm} t\right)
$$

The phase-conjugatıng mirror (at $x=0$ ) couples the two frequencres via the wave equation $[5,17,18]$

$$
\left(\begin{array}{cc}
\mathcal{H}_{0} & \gamma^{*} \\
-\gamma & -\mathcal{H}_{0}
\end{array}\right)\left(\begin{array}{l}
\mathcal{E}_{+} \\
\mathcal{E}_{-}^{*}
\end{array}\right)=\frac{2 \varepsilon \Delta \omega}{\omega_{0}}\left(\begin{array}{l}
\mathcal{E}_{+} \\
\mathcal{E}_{-}^{*}
\end{array}\right)
$$

The complex dimensionless coupling constant $\gamma$ is zero for $x<0$ and for $x>L_{c}$, with $L_{c}$ the length of the nonlinear medium forming the phase-conjugating mirror For $0<x<L_{c}$ it is proportional to the electric fields $\mathcal{E}_{1}, \mathcal{E}_{2}$ of the two pump beams and to the third-order nonlinear suscept1bility $\chi_{3}$

$$
\gamma=-\frac{3}{2 \varepsilon_{0}} \chi_{3} \mathcal{E}_{1}^{*} \mathcal{E}_{2}^{*} \equiv \gamma_{0} e^{\imath \psi}, \quad 0<x<L_{c}
$$

The Helmholtz operator $\mathcal{H}_{0}$ at frequency $\omega_{0}$ is given by

$$
\mathcal{H}_{0}=-k_{0}^{-2} \nabla^{2}-\varepsilon
$$

where $\varepsilon(x, y)$ is the relative dielectric constant of the medium We take $\varepsilon=1$ except in the disordered region $-L<x<0$, where $\varepsilon=1+\delta \varepsilon(x, y)$ The fluctuations $\delta \varepsilon$ lead to scattering with mean free path $l$ We assume $k_{0} l \geqslant 1$, where $k_{0}=\omega_{0} / c$ is the wave number of the light (velocity c) The validity of $\mathrm{Eq}(22)$ requires $\Delta \omega / \omega_{0} \ll 1$ and $|\gamma| \equiv \gamma_{0} \ll 1$ The ratio of these two small parameters

$$
\delta=\frac{2 \Delta \omega}{\gamma_{0} \omega_{0}}
$$

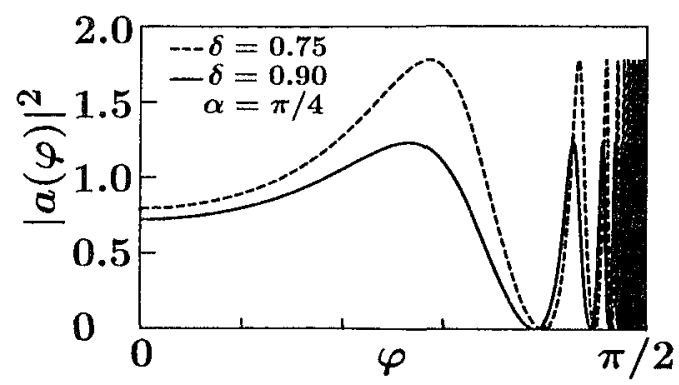

FIG 2 Reflectance of the phase-conjugdting mirror as a function of the angle of incidence, computed from $\mathrm{Eq}(26)$ for two choices of parameters

1s a measure of the degeneracy of the incident and the reflected wave, and can be chosen freely

In the absence of disorder, an incoming plane wave in the direction $(\cos \phi, \sin \phi)$ is retro-reflected in the direction $(-\cos \phi,-\sin \phi)$, with a different frequency and amplitude The scattering matrix for retroreflection is given by $[5,17-$ 20]

$$
\begin{gathered}
\left(\begin{array}{c}
\mathcal{E}_{+} \\
\mathcal{E}_{-}^{*}
\end{array}\right)^{\text {out }}=\left(\begin{array}{cc}
0 & -\imath a(\phi) e^{-\imath \psi} \\
\imath a(\phi) e^{\imath \psi} & 0
\end{array}\right)\left(\begin{array}{l}
\mathcal{E}_{+} \\
\mathcal{E}_{-}^{*}
\end{array}\right)^{\text {In }} \\
a(\phi)=\left[\sqrt{1+\delta^{2}} \operatorname{cotan}\left(\alpha \sqrt{1+\delta^{2}} / \cos \phi\right)+\imath \delta\right]^{-1} \\
\alpha=\frac{1}{2} \gamma_{0} k_{0} L_{c}
\end{gathered}
$$

The cructal difference with Ref [10] is that the reflectance is angle dependent and that the reflection matrix is nonHermitian This implies that not all phases will be canceled in the conjugation process In $\mathrm{F}_{1} \mathrm{~g} 2$ we have plotted the reflectance $|a|^{2}$ as a function of the angle of incidence $\phi$ for $\alpha=\pi / 4$ and two values of $\delta=075$ and 09 The value $\alpha=\pi / 4$ is chosen such that $a=1$ for normal incidence at frequency $\omega_{0}(1 \mathrm{e}$, for $\phi=0, \delta=0)$ The two values of $\delta$ have been chosen such that the angular average of the reflectance,

$$
A=\int_{0}^{\pi / 2} d \phi \cos \phi|a(\phi)|^{2}
$$

1s $>1$ for $\delta=075$ and $<1$ for $\delta=09$ [The $\cos \phi$ werght factor in Eq (27) corresponds to diffusive illumination ] In most of the numencal examples throughout this paper we will use these values of $\alpha$ and $\delta$

\section{PHASE-INCOHERENT SOLUTION}

\section{A. Radiative-transfer theory}

Within the framework of radiative-transfer theory $[13,14]$, the stationary distribution $I(x, y, \phi) \propto|\mathcal{E}|^{2}$ of the light intensity, at frequency $\omega$ and wave vector $(k \cos \phi, k \sin \phi)$, is governed by the Boltzmann equation 


$$
\begin{aligned}
& \left(l \cos \phi \frac{\partial}{\partial x}+l \sin \phi \frac{\partial}{\partial y}\right) I(x, y, \phi) \\
& \quad=-I(x, y, \phi)+\frac{1}{2 \pi} \int_{0}^{2 \pi} d \phi^{\prime} I\left(x, y, \phi^{\prime}\right) .
\end{aligned}
$$

We neglect absorption and assume isotropic scattering in the $x-y$ plane, with mean free path $l$. The phase-conjugating mirror couples the intensities $I_{ \pm}$of light at the two frequencies $\omega_{ \pm}=\omega_{0} \pm \Delta \omega$. We assume that $l$ is independent of frequency. The symmetry of the system implies that $I(x, y, \phi)=I(x,|\phi|)$. In this section we take $\phi \in[0, \pi]$. For each frequency the Boltzmann equation takes the form

$$
\begin{gathered}
l \cos \phi \frac{\partial I_{ \pm}(x, \phi)}{\partial x}=\bar{I}_{ \pm}(x)-I_{ \pm}(x, \phi), \\
\bar{I}_{ \pm}(x)=\frac{1}{\pi} \int_{0}^{\pi} d \phi I_{ \pm}(x, \phi) .
\end{gathered}
$$

Equation (3.2) has to be supplemented by boundary conditions at the two ends $x=-L$ and $x=0$ of the disordered medium. We consider a situation that the system is illuminated at $x=-L$ with diffusive light at frequency $\omega_{+}$, hence

$$
\begin{array}{ll}
I_{+}(-L, \phi)=I_{0} & \text { for } \cos \phi>0, \\
I_{-}(-L, \phi)=0 & \text { for } \cos \phi>0 .
\end{array}
$$

At $x=0$ the light is reflected by the phase-conjugating mirror. The intensity is multiplied by

$$
|a(\phi)|^{2}=\frac{\sin ^{2}\left(\alpha \sqrt{1+\delta^{2}} / \cos \phi\right)}{\delta^{2}+\cos ^{2}\left(\alpha \sqrt{1+\delta^{2}} / \cos \phi\right)},
$$

according to Eq. (2.6). The reflection is accompanied by a change in frequency $\omega_{ \pm} \rightarrow \omega_{\mp}$, so that the boundary condition is

$$
I_{ \pm}(0, \phi)=|a(\phi)|^{2} I_{\mp}(0, \pi-\phi) \text { for } \cos \phi<0 .
$$

The flux $j_{ \pm}$associated with the intensity $I_{ \pm}$is defined by

$$
j_{ \pm}=\int_{0}^{\pi} d \phi \cos \phi I_{ \pm}(x, \phi),
$$

and is independent of $x\left[\partial j_{ \pm} / \partial x=0\right.$ according to Eq. (3.2)]. The reflectance $R_{-}$is defined as the ratio of the outgoing flux at frequency $\omega_{-}$and the incoming flux at frequency $\omega_{+}$,

$$
R_{-}=-j_{-} / I_{0}
$$

The total outgoing flux is $\left(R_{-}+R_{+}\right) I_{0}$, where

$$
R_{+}=1-j_{+} / I_{0}
$$

is the ratio of the outgoing flux and the incoming flux at the same frequency $\omega_{+}$.

\section{B. Neglect of angular correlations}

A simple analytical treatment is possible if the angular correlations between multiple reflections by the disorder and the phase-conjugating mirror are neglected. Here we present this simplified treatment, and in the next subsection we compare with an exact numerical solution of the Boltzmann equation.

We first consider the disordered region by itself. The plane-wave transmission probability $|t(\phi)|^{2}$ is the ratio of transmitted to incident flux when the incident light is a plane wave in the direction $(\cos \phi, \sin \phi)$. The transmission probability $T$ for diffusive illumination is then given by

$$
T=\int_{0}^{\pi / 2} d \phi \cos \phi|t(\phi)|^{2}
$$

such that $T$ is the fraction of the flux incident from a diffusive source which is transmitted through the disordered region. This probability has been calculated in Ref. [21] from the Boltzmann equation (3.2). The result is

$$
T=(1+2 \eta L / \pi l)^{-1},
$$

where $\eta$ is a numerical coefficient which depends weakly on $L / l$. In the ballistic limit $(L / l \rightarrow 0) \eta$ has the value $\pi^{2} / 8$ and in the diffusive limit $(L / l \rightarrow \infty) \eta=1$. In this subsection (but not in the next) we take $\eta=1$ for all $L / l$ for simplicity.

We use Eq. (3.10) to obtain the reflectance $R_{ \pm}$for the case that the disordered medium is backed by a phaseconjugating mirror with reflectance

$$
A=\int_{0}^{\pi / 2} d \phi \frac{\cos \phi \sin ^{2}\left(\alpha \sqrt{1+\delta^{2}} / \cos \phi\right)}{\delta^{2}+\cos ^{2}\left(\alpha \sqrt{1+\delta^{2}} / \cos \phi\right)}
$$

Since $T$ and $A$ are angular averages, we are neglecting angular correlations. The light that comes out at frequency $\omega_{-}$ has been reflected an odd number of times at the mirror. The light that has been reflected once has traversed the medium twice, which leads to a contribution $T^{2} A$ to $R_{-}$. Light that has been reflected three times by the mirror contributes $T^{2} A^{3}(1-T)^{2}$, since it has been reflected two times by the medium (each time with probability $1-T$ ). Summing all contributions, one finds

$$
\begin{aligned}
R_{-} & =T^{2} A+T^{2} A^{3}(1-T)^{2}+T^{2} A^{5}(1-T)^{4}+\cdots \\
& =\frac{T^{2} A}{1-(1-T)^{2} A^{2}}
\end{aligned}
$$

Light that comes out at frequency $\omega_{+}$has been reflected an even number of times at the mirror. Zero reflections by the mirror contributes $1-T$ to $R_{+}$, two reflections contributes $T^{2} A^{2}(1-T)$, and four reflections $T^{2} A^{4}(1-T)^{3}$. Summing the series, one finds

$$
R_{+}=1-T+\frac{T^{2}(1-T) A^{2}}{1-(1-T)^{2} A^{2}} .
$$

The geometric series leading to Eq. (3.12) diverges if $(1-T) A \geqslant 1$. This indicates that there is only a stationary solution to the Boltzmann equation if both the gain at the 
mirror and the scattering in the medium are sufficiently weak. If $A$ is increased at fixed $\alpha=\pi / 4$ by reducing $\delta$, the reflectances $R_{ \pm}$diverge when $\delta=\delta_{c}$. (This divergence is preempted by depletion of the pump beams in the phaseconjugating mirror, which we do not describe.) In the approximation of this subsection, $\delta_{c}$ is determined by $(1-T) A=1$, or $L / l=\frac{1}{2} \pi(A-1)^{-1}$. In the ballistic limit, $T=1$ and $A<\infty$ for any $\delta>0$. In the diffusive limit, $T=0$ and $A=1$ for $\delta=0.78$. Hence, $\delta_{c}$ increases from 0 to 0.78 as $L / l$ increases from 0 to $\infty$.

\section{Exact solution of the Boltzmann equation}

The Boltzmann equation (3.2) can be solved exactly, without neglect of angular correlations, by adapting the method of Ref. [21] to an angle-dependent boundary condition. We first rewrite Eq. (3.2) as

$$
\frac{\partial}{\partial x} e^{x / l \cos \phi} I_{ \pm}(x, \phi)=\frac{1}{l \cos \phi} e^{x / l \cos \phi} \bar{I}_{ \pm}(x),
$$

and then integrate once over $x$, using the boundary conditions (3.3) and (3.5). The result is

$$
\begin{aligned}
& I_{+}(x, \phi)=\int_{-L}^{x} \frac{d x^{\prime}}{l \cos \phi} e^{-\left(x-x^{\prime}\right) / l \cos \phi} \bar{I}_{+}\left(x^{\prime}\right) \\
& +I_{0} e^{-(L+x) / l \cos \phi} \text { for } \cos \phi>0 \text {, } \\
& \bar{I}_{--}(x)=\int_{-L}^{0} d x^{\prime} M_{1}\left(x, x^{\prime}\right) \bar{I}_{--}\left(x^{\prime}\right) \\
& +\int_{-L}^{0} d x^{\prime} M_{2}\left(x, x^{\prime}\right) \bar{I}_{+}\left(x^{\prime}\right)+Q_{2}(x) I_{0} .
\end{aligned}
$$$$
I_{+}(x, \phi)=-\int_{x}^{0} \frac{d x^{\prime}}{l \cos \phi} e^{-\left(x-x^{\prime}\right) / / \cos \phi} \bar{I}_{+}\left(x^{\prime}\right)
$$$$
+e^{-x / l \cos \phi}|a(\phi)|^{2} I_{-}(0, \pi-\phi)
$$$$
\text { for } \cos \phi<0,(3.14 b)
$$$$
I_{-}(x, \phi)=\int_{-L}^{x} \frac{d x^{\prime}}{l \cos \phi} e^{-\left(x-x^{\prime}\right) / l \cos \phi} \bar{I}_{-}\left(x^{\prime}\right)
$$$$
\text { for } \cos \phi>0,(3.14 \mathrm{c})
$$$$
I_{-}(x, \phi)=-\int_{x}^{0} \frac{d x^{\prime}}{l \cos \phi} e^{-\left(x-x^{\prime}\right) / l \cos \phi} \bar{I}_{-}\left(x^{\prime}\right)
$$$$
+e^{-x / l \cos \phi}|a(\phi)|^{2} I_{+}(0, \pi-\phi)
$$$$
\text { for } \cos \phi<0 \text {. }
$$

Substitution of Eqs. (3.14c) and (3.14a) into, respectively, Eqs. (3.14b) and (3.14d) yields

$$
\begin{aligned}
I_{+}(x, \phi)= & \int_{x}^{0} \frac{d x^{\prime}}{l|\cos \phi|} e^{-\left(x^{\prime}-x\right) / l|\cos \phi|} \bar{I}_{+}\left(x^{\prime}\right) \\
& +e^{x / l|\cos \phi|}|a(\phi)|^{2} \\
& \times \int_{-L}^{0} \frac{d x^{\prime}}{l|\cos \phi|} e^{x^{\prime} / l|\cos \phi|} \bar{I}_{-}\left(x^{\prime}\right)
\end{aligned}
$$

for $\cos \phi<0,(3.15 \mathrm{a})$

$$
\begin{aligned}
I_{-}(x, \phi)= & \int_{x}^{0} \frac{d x^{\prime}}{l|\cos \phi|} e^{-\left(r^{\prime}-x\right) / l|\cos \phi|} \bar{I}_{-}\left(x^{\prime}\right) \\
& +e^{x / l|\cos \phi|}|a(\phi)|^{2} \times\left(I_{0} e^{-L / l|\cos \phi|}\right. \\
& \left.+\int_{-L}^{0} \frac{d x^{\prime}}{l|\cos \phi|} e^{x^{\prime} l|\cos \phi|} \bar{I}_{+}\left(x^{\prime}\right)\right)
\end{aligned}
$$

for $\cos \phi<0 .(3.15 b)$

Finally, integration over $\phi$ leads to two coupled integral equations for the average intensities,

$$
\begin{aligned}
\bar{I}_{+}(x)= & \int_{-L}^{0} d x^{\prime} M_{1}\left(x, x^{\prime}\right) \bar{I}_{+}\left(x^{\prime}\right) \\
& +\int_{-L}^{0} d x^{\prime} M_{2}\left(x, x^{\prime}\right) \bar{I}_{-}\left(x^{\prime}\right)+Q_{1}(x) I_{0},
\end{aligned}
$$

We have defined the following kernels and source terms:

$$
\begin{gathered}
M_{1}\left(x, x^{\prime}\right)=\frac{1}{\pi} \int_{0}^{\pi / 2} \frac{d \phi}{l \cos \phi} e^{-\left|x-x^{\prime}\right| / l \cos \phi} \\
=\frac{1}{\pi l} K_{0}\left(\left|x-x^{\prime}\right| / l\right), \\
M_{2}\left(x, x^{\prime}\right)=\frac{1}{\pi} \int_{0}^{\pi / 2} \frac{d \phi}{l \cos \phi} e^{\left(x+\lambda^{\prime}\right) / l \cos \phi}|a(\phi)|^{2}, \\
Q_{1}(x)=\frac{1}{\pi} \int_{0}^{\pi / 2} d \phi e^{-(L+x) / l \cos \phi}, \\
Q_{2}(x)=\frac{1}{\pi} \int_{0}^{\pi / 2} d \phi e^{-(L-x) / l \cos \phi}|a(\phi)|^{2},
\end{gathered}
$$

where $K_{0}$ is a Bessel function.

Equation (3.16) is the analog for the present problem involving two coupled frequencies of the Schwarzschild-Milne equation in the theory of radiative transfer $[13,14]$. We have solved it numerically by discretizing with respect to $x$ so that the integral equation becomes a matrix equation. From the average intensities $\bar{I}_{ \pm}(x)$ one finds the intensities $I_{ \pm}(x, \phi)$ using Eqs. (3.14) and (3.15). The reflectances $R_{ \pm}$then follow from Eqs. (3.6)-(3.8). For numerical stability we have imposed a cutoff on the rapidly oscillating function $a(\phi)$ at grazing incidence, by setting $a(\phi)=0$ for $0.497 \pi<\phi<\frac{1}{2} \pi$. 

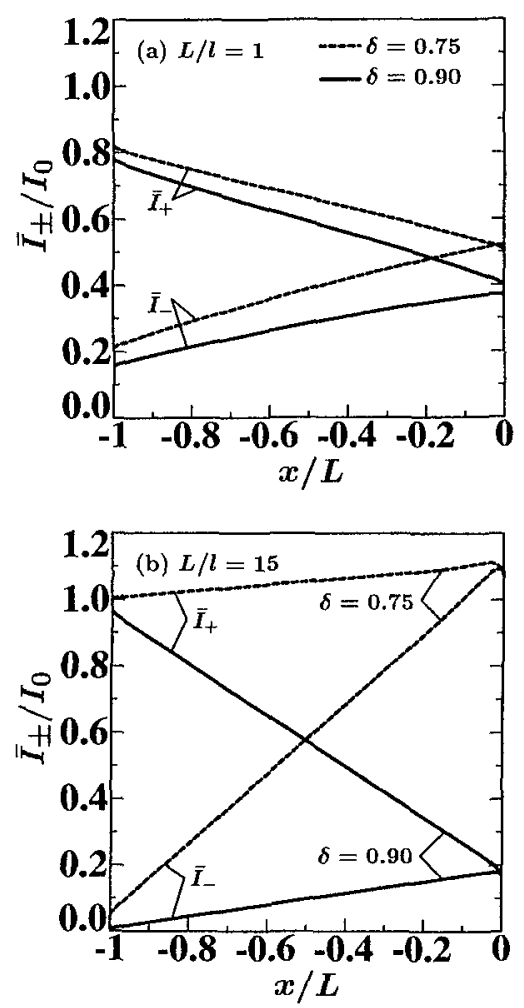

FIG. 3. Intensity profiles in the disordered medium, computed from the exact numerical solution of the Boltzmann equation, for $\alpha=\pi / 4$ and two values of $\delta$. (a) is for a nearly ballistic system $(L / l=1)$, (b) is for a diffusive system $(L / l=15)$.

In Figs. 3 and 4 we show results for $\bar{I}_{ \pm}(x)$ and $R_{ \pm}$for $\alpha=\pi / 4$ and $\delta=0.75$ and 0.9 . For $\delta=0.75$ there is an effective gain at the mirror $(A>1)$, while for $\delta=0.9$ there is an effective loss $(A<1)$. For an ordinary mirror one can show that $\bar{I}_{ \pm}(0)=\frac{1}{2} I_{0}$. Instead, we find that $\bar{I}_{-}(0)>\bar{I}_{+}(0)>\frac{1}{2} I_{0}$ for $\delta=0.75$, indicating gain, and $\bar{I}_{-}(0)<\bar{I}_{+}(0)<\frac{1}{2} I_{0}$ for $\delta=0.9$, indicating loss. In each case the density profiles are approximately linear in the bulk, with some bending near the boundaries at $x=-L$ and $x=0$. For $\delta=0.75$, both $R_{-}$and $R_{+}$diverge when $L / l=28$, while for $\delta=0.9$ no such diver-

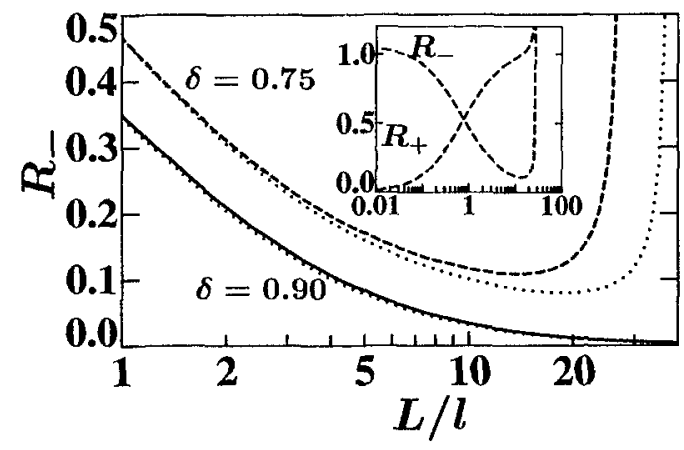

FIG. 4. Reflectance $R_{-}$as a function of $L / l$, computed from the exact solution of the Boltzmann equation for $\alpha=\pi / 4$ and $\delta=0.75$ (dashed curve), $\delta=090$ (solid curve). The dotted curves are the approximate result (3.12a), in which angular correlations are neglected. The inset shows the exact reflectances $R_{ \pm}$for $\delta=0.75$, over a broader range of $L / l$ (logarithmic scale). For $\delta=0.75$ the reflectances diverge at $L / l=28$. No divergence occurs for $\delta=0.90$.

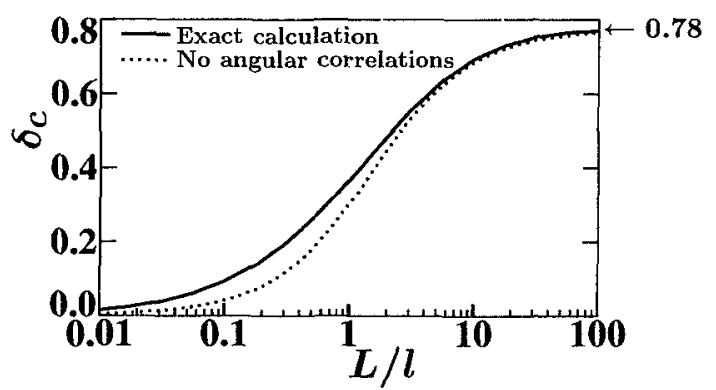

FIG. 5. A stationary solution to the Boltzmann equation requires $\delta>\delta_{c}$. The solid curve is the exact result for $\delta_{c}$ (at fixed $\alpha=\pi / 4$, as function of $L / l)$, the dotted curve follows from Eq. (3.12), obtained by neglecting angular correlations.

gence occurs. As discussed earlier, the divergence indicates that for $\delta=0.75$ and $L / l>28$ there is no stationary solution to the Boltzmann equation. For fixed $L / l$ and $\alpha$, the divergence of $R_{ \pm}$occurs at a critical value $\delta_{c}$, such that a stationary solution requires $\delta>\delta_{c}$. The dependence of $\delta_{c}$ on $L / l$ at fixed $\alpha=\pi / 4$ is plotted in Fig. 5 .

In Figs. 4 and 5 we also compare the exact numerical solution of the Boltzmann equation of this subsection with the approximate analytical solution (3.12) of the preceding subsection. As one can see, the agreement with the exact results is quite good.

\section{PHASE-COHERENT SOLUTION}

\section{A. Scattering matrices}

We now turn to a phase-coherent description of the scattering problem. To define finite-dimensional scattering matrices we embed the disordered medium in a waveguide (width $W$ ), containing $N_{ \pm}=\operatorname{Int}\left(\omega_{ \pm} W / c \pi\right) \gg 1$ propagating modes at frequency $\omega_{ \pm}$. $\overrightarrow{\mathrm{A}}$ basis of scattering states consists of the complex fields

$$
\begin{aligned}
& E_{ \pm, n}^{>}(x, y, t)=k_{ \pm, n}^{-1 / 2} \sin \left(\frac{n \pi y}{W}\right) \exp \left(i k_{ \pm, n} x-i \omega_{ \pm} t\right), \\
& E_{ \pm, n}^{<}(x, y, t)=k_{ \pm, n}^{-1 / 2} \sin \left(\frac{n \pi y}{W}\right) \exp \left(-i k_{ \pm, n} x-i \omega_{ \pm} t\right) .
\end{aligned}
$$

Here $n=1,2, \ldots, N_{ \pm}$is the mode index and the superscript $>(<)$ indicates a wave moving to the right (left), with frequency $\omega_{ \pm}=\omega_{0} \pm \Delta \omega$ and wave number

$$
k_{ \pm, n}=\left(\omega_{ \pm}^{2} / c^{2}-n^{2} \pi^{2} / W^{2}\right)^{1 / 2} .
$$

The normalization in Eq. (4.1) has been chosen such that each wave carries the same flux.

With respect to the basis (4.1), incoming and outgoing waves are decomposed as

$$
\begin{aligned}
& E^{\mathrm{in}}=\sum_{n=1}^{N_{+}} u_{+, n} E_{+, n}^{>}+\sum_{n=1}^{N_{-}} u_{-, n} E_{-, n}^{>0}, \\
& E^{\text {out }}=\sum_{n=1}^{N_{+}} v_{+, n} E_{+, n}^{<}+\sum_{n=1}^{N_{-}} v_{-, n} E_{-, n}^{<} .
\end{aligned}
$$


The complex coefficients are combined into two vectors

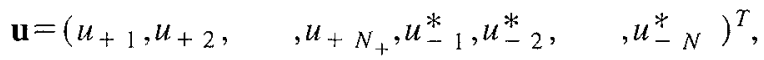

$$
\begin{aligned}
& \mathbf{v}=\left(v_{+1}, v_{+2}, \quad, v+N_{+}, v_{-1}^{*}, v_{-2}^{*}, \quad, v_{-N}^{*}\right)^{T}
\end{aligned}
$$

The reflection matrix $\mathbf{r}$ relates $\mathbf{u}$ to $\mathbf{v}$,

$$
\mathbf{v}=\mathbf{r u}, \quad \mathbf{r}=\left(\begin{array}{ll}
\mathbf{r}_{++} & \mathbf{r}_{+-} \\
\mathbf{r}_{-+} & \mathbf{r}_{--}
\end{array}\right)
$$

The dimension of $\mathbf{r}$ is $\left(N_{+}+N_{-}\right) \times\left(N_{+}+N_{-}\right)$, the submatrices $\mathbf{r}_{+}$have dimensions $N_{+} \times N_{ \pm}$For $\Delta \omega \ll \omega_{0}$ we may neglect the difference between $N_{+}$and $N_{-}$and replace both by $N=\operatorname{Int}\left(k_{0} W / \pi\right)$

In the absence of disorder the reflection matrix is entirely determined by the phase-conjugating mirror,

$$
\begin{gathered}
\mathbf{r}_{\mathrm{PCM}}=\left(\begin{array}{cc}
0 & -\imath \mathbf{a} e^{-\imath \psi} \\
\imath \mathbf{a} e^{i \psi} & 0
\end{array}\right), \\
a_{m n}=a\left(\phi_{n}\right) \delta_{m n}, \quad \phi_{n}=\arcsin \left(n \pi / k_{0} W\right)
\end{gathered}
$$

The elements of the $N \times N$ diagonal matrix a are obtaned from Eq (26) upon substitution of $\phi$ by $\phi_{n}$, being the angle of incidence associated with mode $n$ (The difference in angle between the two frequencies $\omega_{+}$and $\omega_{-}$can be neglected if $\Delta \omega \ll \omega_{0}$ ) The angular average (2 7) of the reflectance corresponds to the modal average

$$
A=\frac{1}{N} \operatorname{Tr} \mathbf{a a}^{\dagger}
$$

In the limit $N \rightarrow \infty$ the two averages are identical

The disordered medium in front of the phase-conjugating mirror does not couple $\omega_{+}$and $\omega_{-}$Its scatternng properties at frequency $\omega$ are described by two $N \times N$ transmission matrices $\mathbf{t}_{21}(\omega)$ and $\mathbf{t}_{12}(\omega)$ (transmission from left to right and from right to left) plus two $N \times N$ reflection matrices $\mathbf{r}_{11}(\omega)$ and $\mathbf{r}_{22}(\omega)$ (reflection from left to left and from right to right) Taken together, these four matrices constıtute a $2 N \times 2 N$ scattering matrix

$$
\mathbf{S}_{\mathrm{d} 1 \text { order }}(\omega)=\left(\begin{array}{ll}
\mathbf{r}_{11}(\omega) & \mathbf{t}_{12}(\omega) \\
\mathbf{t}_{21}(\omega) & \mathbf{r}_{22}(\omega)
\end{array}\right)
$$

which is unitary (because of flux conservation) and symmetric (because of time-reversal invariance) It is simple algebra to express the scattering matrix $\mathbf{r}$ of the entıre system in terms of the scattering matrices $\mathbf{r}_{\mathrm{PCM}}$ and $\boldsymbol{S}_{\mathrm{d} \text { solder }}$ of the phase-conjugating mirror and the disordered region separately The result is

$$
\begin{aligned}
\mathbf{r}_{++}= & \mathbf{r}_{11}\left(\omega_{+}\right)+\mathbf{t}_{12}\left(\omega_{+}\right) \mathbf{a r}_{22}^{*}\left(\omega_{-}\right) \\
& \times \mathbf{a}\left[1-\mathbf{r}_{22}\left(\omega_{+}\right) \mathbf{a r}_{22}^{*}\left(\omega_{-}\right) \mathbf{a}\right]^{-1} \mathbf{t}_{21}\left(\omega_{+}\right), \\
\mathbf{r}_{-}= & \mathbf{r}_{11}^{*}\left(\omega_{-}\right)+\mathbf{t}_{12}^{*}\left(\omega_{-}\right) \mathbf{a} \mathbf{r}_{22}\left(\omega_{+}\right) \\
& \times \mathbf{a}\left[1-\mathbf{r}_{22}^{*}\left(\omega_{-}\right) \mathbf{a r}_{22}\left(\omega_{+}\right) \mathbf{a}\right]^{-1} \mathbf{t}_{21}^{*}\left(\omega_{-}\right),
\end{aligned}
$$

$$
\mathbf{r}_{-+}=\imath e^{\iota \psi} \mathbf{t}_{12}^{*}\left(\omega_{-}\right) \mathbf{a}\left[1-\mathbf{r}_{22}\left(\omega_{+}\right) \mathbf{a r}_{22}^{*}\left(\omega_{-}\right) \mathbf{a}\right]^{-1} \mathbf{t}_{21}\left(\omega_{+}\right)
$$

$$
\mathbf{r}_{+-}=-\imath e^{-\imath \psi} \mathbf{t}_{12}\left(\omega_{+}\right) \mathbf{a}\left[1-\mathbf{r}_{22}^{*}\left(\omega_{-}\right) \mathbf{a r}_{22}\left(\omega_{+}\right) \mathbf{a}\right]^{-1} \mathbf{t}_{21}^{*}\left(\omega_{--}\right)
$$

We seek the reflectances

$$
R_{-}=\frac{1}{N_{+}} \operatorname{Tr} \mathbf{r}_{-+} \mathbf{r}_{-+}^{\dagger}, \quad R_{+}=\frac{1}{N_{+}} \operatorname{Tr} \mathbf{r}_{++} \mathbf{r}_{++}^{\dagger},
$$

averaged over the disorder We will do this analytically, using random-matrix theory [22], and numerically, using the recursive Green tunction technique [23] We consider two different regimes, depending on the relative magnitude of $\Delta \omega$ and $1 / \tau_{\text {dwell }}$, where $\tau_{\text {dwell }} \simeq L^{2} / c l$ is the mean dwell time of a photon in the disordered medium If $\tau_{\text {dwell }} \Delta \omega \ll 1$ the difference between $\mathbf{S}_{\text {disoider }}\left(\omega_{+}\right)$and $\mathbf{S}_{\text {disorder }}\left(\omega_{-}\right)$is insignificant, because the phase shifts accumulated in a time $\tau_{\text {dwell }}$ are approximately the same for frequencies $\omega_{+}$and $\omega_{-}$We call this the coherent regime If $\tau_{\mathrm{dwel1}} \Delta \omega \gg 1$, on the contrary, phase shifts at $\omega_{+}$and $\omega_{-}$are essentially uncorrelated, so that $\mathbf{S}_{\mathrm{d} 1 \text { sorder }}\left(\omega_{+}\right)$and $\mathbf{S}_{\mathrm{d} \text { sorder }}\left(\omega_{-}\right)$are independent We call this the incoherent regime

\section{B. Random-matrix theory}

Without loss of generality the reflection and transmission matrices of the disordered region can be decomposed as [22]

$$
\begin{gathered}
\mathbf{r}_{11}\left(\omega_{ \pm}\right)=\iota \mathbf{V}_{ \pm} \sqrt{1-\mathbf{T}_{ \pm}} \mathbf{V}_{ \pm}^{T}, \\
\mathbf{r}_{22}\left(\omega_{ \pm}\right)={ }_{l} \mathbf{U}_{ \pm} \sqrt{1-\mathbf{T}_{ \pm}} \mathbf{U}_{ \pm}^{T}, \\
\mathbf{t}_{12}\left(\omega_{ \pm}\right)=\mathbf{V}_{ \pm} \sqrt{\mathbf{T}_{ \pm}} \mathbf{U}_{ \pm}^{T}, \mathbf{t}_{21}\left(\omega_{ \pm}\right)=\mathbf{U}_{ \pm} \sqrt{\mathbf{T}_{ \pm}} \mathbf{V}_{ \pm}^{T}
\end{gathered}
$$

Here $\mathbf{U}_{ \pm}$and $\mathbf{V}_{ \pm}$are $N \times N$ unitary matrices (we take $N_{+}=N_{-}=N$ in this subsection) and $\mathbf{T}_{ \pm}$is a diagonal mattix with the transmission ergenvalues $\tau_{ \pm n} \in[0,1]$ on the diagonal The subscript \pm refers to the two frequencies $\omega_{+}$and $\omega_{\ldots}$ In this so-called "polar decomposition" the reflectances $R_{ \pm}$take the form

$$
\begin{aligned}
& R=\frac{1}{N} \operatorname{Tr} \mathbf{T}_{-} \mathbf{\Omega}\left(1-\sqrt{1-\mathbf{T}_{+}} \mathbf{\Omega}^{T} \sqrt{1-\mathbf{T}_{-}} \mathbf{\Omega}\right)^{-1} \mathbf{T}_{+} \\
& \times\left(1-\boldsymbol{\Omega}^{\dagger} \sqrt{1-\mathbf{T}} \boldsymbol{\Omega}^{k} \sqrt{1-\mathbf{T}_{+}}\right)^{-1} \mathbf{\Omega}^{\dagger}, \\
& R_{+}=\frac{1}{N} \operatorname{Tr}\left(1-\mathbf{T}_{+}\right)+\frac{1}{N} \operatorname{Tr} \mathbf{T}_{+} \boldsymbol{\Omega}^{T} \sqrt{1-\mathbf{T}_{-}} \\
& \times \mathbf{\Omega}\left(1-\sqrt{1-\mathbf{T}_{+}} \mathbf{\Omega}^{T} \sqrt{1-\mathbf{T}_{-}} \boldsymbol{\Omega}\right)^{-1} \mathbf{T}_{+}\left(1-\mathbf{\Omega}^{\dagger}\right. \\
& \left.\times \sqrt{1-\mathbf{T}_{-}} \Omega^{*} \sqrt{1-\mathbf{T}_{+}}\right)^{-1} \mathbf{\Omega}^{i} \sqrt{1-\mathbf{T}_{-}} \mathbf{\Omega}^{*} \\
& -\frac{1}{N} \operatorname{Tr} \mathbf{T}_{+} \sqrt{1-\mathbf{T}_{+}}\left(1-\mathbf{\Omega}^{\mathrm{\top}} \sqrt{1-\mathbf{T}_{-}} \mathbf{\Omega}^{*} \sqrt{1-\mathbf{T}_{+}}\right)^{-1} \\
& \times \mathbf{\Omega}^{\dagger} \sqrt{1-\mathbf{T}_{-}} \mathbf{\Omega}^{*}-\frac{1}{N} \operatorname{Tr} \mathbf{T}_{+} \sqrt{1-\mathbf{T}_{+}} \boldsymbol{\Omega}^{T} \sqrt{1-\mathbf{T}_{-}} \\
& \times \mathbf{\Omega}\left(1-\sqrt{1-\mathbf{T}_{+}} \mathbf{\Omega}^{r} \sqrt{1-\mathbf{T}_{-}} \mathbf{\Omega}\right)^{-1},
\end{aligned}
$$




$$
\mathbf{\Omega}=\mathbf{U}_{-}^{\dagger} \mathbf{a} \mathbf{U}_{+} .
$$

To compute the averages $\left\langle R_{ \pm}\right\rangle$analytically in the large- $N$ limit we make the isotropy approximation [22] that the matrices $\mathbf{U}_{ \pm}$and $\mathbf{V}_{ \pm}$are uniformly distributed over the unitary group $\mathcal{U}(N)$. This approximation corresponds to the neglect of angular correlations in the radiative-transfer theory ( $\mathrm{Sec}$. III B). For $\tau_{\text {dwell }} \Delta \omega \ll 1$ we may identify $\mathbf{U}_{+}=\mathbf{U}_{-}$and $\mathbf{V}_{+}=\mathbf{V}_{-}$. For $\tau_{\text {dwell }} \Delta \omega \gg 1$ we may assume that $\mathbf{U}_{+}, \mathbf{U}_{-}$, $\mathbf{V}_{+}$, and $\mathbf{V}_{-}$are all independent. In each case the integration $\int d \mathbf{U} f(\mathbf{U})$ over $\mathcal{U}(N)$ with $N \gg 1$ can be done using the large$N$ expansion of Ref. [24]. The remaining average over $\tau_{ \pm, n}$ can be done using the known density $\rho(\tau)$ of the transmission eigenvalues in a disordered medium [22].

The calculation is easiest in the incoherent regime ( $\tau_{\text {dwell }} \Delta \omega \gg 1$ ). The integration over $\mathcal{U}(N)$ can be carried out using the formula [24]

$$
\begin{aligned}
\int & d \mathbf{U} \int d \mathbf{V} \frac{1}{N} \operatorname{Tr}\left(\mathbf{A}_{1} \mathbf{U} \mathbf{A}_{2} \mathbf{V} \mathbf{A}_{3} \mathbf{U} \cdots \mathbf{A}_{p}\right) \\
& \times\left(\mathbf{B}_{1} \mathbf{U} \mathbf{B}_{2} \mathbf{V} \mathbf{B}_{3} \mathbf{U} \cdots \mathbf{B}_{q}\right)^{\dagger} \\
= & \delta_{p q} N^{-p} \prod_{i=1}^{p} \operatorname{Tr} \mathbf{A}_{i} \mathbf{B}_{i}^{\dagger}+O\left(N^{-p-1}\right) .
\end{aligned}
$$

To apply this formula we expand the inverse matrices in Eq. (4.12) in a power series in $\mathbf{U}_{ \pm}$and integrate term by term over the independent matrices $\mathbf{U}_{+}$and $\mathbf{U}_{-}$. The result is, to leading order in $N$,

$$
\begin{aligned}
\int d \mathbf{U}_{-} \int d \mathbf{U}_{+} R_{-} & =\sum_{p=0}^{\infty} T_{-} T_{+} A^{2 p+1}\left(1-T_{-}\right)^{p}\left(1-T_{+}\right)^{p} \\
& =\frac{T_{-} T_{+} A}{1-\left(1-T_{-}\right)\left(1-T_{+}\right) A^{2}}, \\
\int d \mathbf{U}_{-} \int d \mathbf{U}_{+} R_{+} & =1-T_{+}+\frac{T_{+}^{2}\left(1-T_{-}\right) A^{2}}{1-\left(1-T_{-}\right)\left(1-T_{+}\right) A^{2}},
\end{aligned}
$$

where we have defined the modal average

$$
T_{ \pm}=\frac{1}{N} \operatorname{Tr} \mathbf{T}_{ \pm}=\frac{1}{N} \sum_{n=1}^{N} \tau_{ \pm, n}
$$

The modal average $A$ was defined in Eq. (4.7). The quantities $T_{ \pm}$still depend on the configuration of the scatterers, but the fluctuations around the average $\left\langle T_{ \pm}\right\rangle$are smaller by an order $1 / N$ than the average itself. Moreover, the average $\left\langle T_{ \pm}\right\rangle$ equals the transmission probability $T$ from radiative-transfer theory, Eq. (3.10), again up to corrections of order $1 / N$. Replacing $T_{ \pm}$in Eq. (4.14) by $T$ we obtain

$$
\begin{gathered}
\left\langle R_{-}\right\rangle=\frac{T^{2} A}{1-(1-T)^{2} A^{2}}, \\
\left\langle R_{+}\right\rangle=1-T+\frac{T^{2}(1-T) A^{2}}{1-(1-T)^{2} A^{2}},
\end{gathered}
$$

which is the result (3.12) of radiative-transfer theory with neglect of angular correlations. The conclusion is that in the incoherent regime phase coherence has no effect on the reflectance of the system to leading order in $N$.

The situation is entirely different in the coherent regime ( $\tau_{\text {dwell }} \Delta \omega \ll 1$ ). To see the difference it is instructive to first consider the simplified model that the matrix $a_{m n}=a_{0} \delta_{m n}$ is proportional to the unit matrix (a scalar). Because $\mathbf{U}_{-}=\mathbf{U}_{+}$ for $\tau_{\text {dwell }} \Delta \omega \ll 1$, we then have $\Omega_{m n}=a_{0} \delta_{m n}$. There is therefore no average over $\mathcal{U}(N)$ to perform. We only have to average over one set of transmission eigenvalues $\tau_{+, n}=\tau_{-, n} \equiv \tau_{n}$. This average amounts to the integrals

$$
\begin{gathered}
\left\langle R_{-}\right\rangle=\frac{1}{N} \int_{0}^{1} d \tau \rho(\tau) \frac{\left|a_{0}\right|^{2} \tau^{2}}{\left|1-a_{0}^{2}+a_{0}^{2} \tau\right|^{2}}, \\
\left\langle R_{+}\right\rangle=1-\frac{1}{N} \int_{0}^{1} d \tau \rho(\tau) \frac{\tau-\left|a_{0}\right|^{4} \tau(1-\tau)}{\left|1-a_{0}^{2}+a_{0}^{2} \tau\right|^{2}} .
\end{gathered}
$$

The density $\rho(\tau)$ for $l \leqslant L \ll N l$ is given by [22]

$$
\rho(\tau)=\frac{N}{2(s+1)} \frac{1}{\tau \sqrt{1-\tau}}+O(s+1)^{-4}, \quad s=\frac{2 L}{\pi l} .
$$

The density has a cutoff for exponentially small $\tau$, which is irrelevant for $\left\langle R_{ \pm}\right\rangle$if $a_{0}^{2} \neq 1$. Substitution of Eq. (4.18) into Eq. (4.17) yields the average reflectances

$$
\begin{gathered}
\left\langle R_{-}\right\rangle=2 T \operatorname{Re} \frac{a_{0}^{*}\left(a_{0}^{2}-1\right)}{a_{0}^{2}-a_{0}^{* 2}} \operatorname{artanh} a_{0}, \\
\left\langle R_{+}\right\rangle=1-2 T \operatorname{Re} \frac{a_{0}^{*}\left(a_{0}^{2}-1\right)}{a_{0}^{2}-a_{0}^{* 2}} \operatorname{artanh} a_{0}^{*},
\end{gathered}
$$

where $T$ is again the transmission probability (3.10) from radiative-transfer theory. Both quantities have a smooth $L$ dependence, with $\left\langle R_{-}\right\rangle$decreasing monotonically $\propto 1 / L$. In contrast, radiative-transfer theory predicts a nonmonotonic $L$ dependence for $A>1$, leading to a divergence at some $L$. For $A<1$, radiative-transfer theory predicts a quadratic decrease of $\left\langle R_{-}\right\rangle \propto 1 / L^{2}$, for large $L$. The conclusion is that, in the coherent regime, phase coherence modifies the reflectance of the phase-conjugating mirror to leading order in $N$.

The result (4.19) was obtained for the simplified model of a scalar reflection matrix a. The true a in Eq. (4.6) is diagonal but not a scalar. This complicates the calculation because $\boldsymbol{\Omega}=\mathbf{U}_{-}^{\dagger} \mathbf{a} \mathbf{U}_{+}$then needs to be averaged over $\mathcal{U}(N)$ even though $\mathbf{U}_{-}=\mathbf{U}_{+}$. The calculation is outlined in the Appendix. The complete result is a complicated function of $L / l$ (plotted in Fig. 6). For $L / l \gg 1$ the result takes the form of Eq. (4.19), where now $a_{0}$ is to be determined from the equation

$$
\frac{1}{N} \operatorname{Tr} \frac{\mathbf{a}}{1-a_{0} \mathbf{a}}=\frac{a_{0}}{1-a_{0}^{2}}
$$

In the limit $N \rightarrow \infty$ this becomes an integral equation for $a_{0}$, 


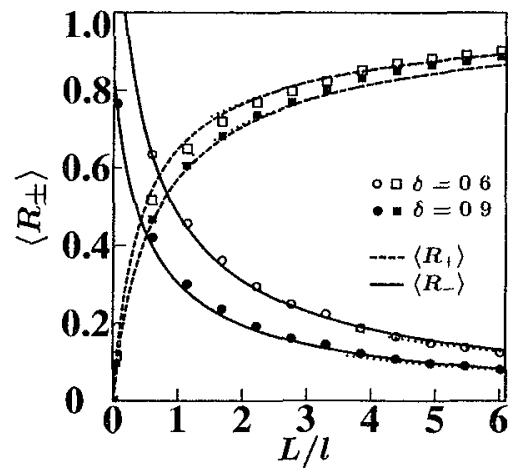

FIG 6 Average reffectances $\left\langle R_{+}\right\rangle$as a function of $L / l$ for $\alpha=\pi / 4$ and $\delta=06,09$, in the coherent regime The full curves ale the analytical results for $N \gg 1$, computed from Eq (A18) The dot ted curves are the large $L / l$ limit given by Eqs (4 19) and (4 21) Data points are results from numerical simulations

$$
\int_{0}^{\pi / 2} d \phi \frac{\cos \phi a(\phi)}{1-a_{0} a(\phi)}=\frac{a_{0}}{1-a_{0}^{2}}
$$

where $a(\phi)$ is given by Eq (26) As shown in Fig 6, the large- $L$ asymptote (419), (420) is close to the complete result for $L \gtrsim l$ In the limit $\delta \rightarrow 0$ the solution to Eq (421) is given by $a_{0}=1284-00133 i$, for $\alpha=\pi / 4$ The corresponding reflectances (for $L \geqslant l$ ) are $\left\langle R_{-}\right\rangle=611 l / L$, and $\left\langle R_{+}\right\rangle=1+577 l / L$

To make contact with the work on wave-front reconstructıon $[11,12]$, we consider also the case of plane-wave-rather than diffusive-illumination A plane wave incident at fiequency $\omega_{+}$in mode $n$ is reflected into modes $m=1,2, \quad, N$ at frequency $\omega_{ \pm}$with probability $\left\langle\left|\left(\mathbf{r}_{ \pm+}\right)_{m n}\right|^{2}\right\rangle$ The calculation of this probability proceeds similarly as the calculation of $R_{\text {_ }} \quad$ (See Ref [25] for the analogous calculation in the case of Andreev reflection ) Using Eqs (4 9)-(4 12) we can write

$$
\begin{gathered}
\mathbf{r}_{-+}=l e^{l \psi} \mathbf{V}^{\nu} \mathbf{O V}_{+}^{T}, \\
\mathbf{O}=\sqrt{\mathbf{T}_{-}} \boldsymbol{\Omega}\left(1-\sqrt{1-\mathbf{T}_{+}} \boldsymbol{\Omega}^{T} \sqrt{1-\mathbf{T}_{-}} \boldsymbol{\Omega}\right)^{-1} \sqrt{\mathbf{T}_{+}}
\end{gathered}
$$

For the coherent regime, we may again identify $\mathbf{V}_{+}=\mathbf{V}_{-}=\mathbf{V}$ The integration over $\mathcal{U}(N)$ can be performed using [24]

$$
\begin{aligned}
\int d \mathbf{V} V_{n k} V_{m j} V_{n l}^{*} V_{m l}^{+}= & \frac{1}{N^{2}-1}\left(\delta_{l k} \delta_{\jmath l}+\delta_{m n} \delta_{k l} \delta_{j l}\right) \\
& -\frac{1}{N^{3}-N}\left(\delta_{k l} \delta_{\jmath l}+\delta_{m n} \delta_{l k} \delta_{\jmath l}\right)
\end{aligned}
$$

We then find

$$
\int d \mathbf{V}\left|\left(\mathbf{r}_{-+}\right)_{m n}\right|^{2}=\frac{1+\delta_{m n}}{N+1} R+\frac{N \delta_{m n}-1}{N^{3}-N} \sum_{i \neq j} \mathbf{O}_{l i} \mathbf{O}_{J}^{*}
$$

In the limit of large $N$ we can write $\Sigma_{l \neq j} \mathbf{O}_{l l} \mathbf{O}_{J j}^{4}=\left|N^{-1} \operatorname{Tr} \mathbf{O}\right|^{2}$ In the same way as before, for $L \geqslant l$, this trace can be expressed in terms of $a_{0}$, where $a_{0}$ can be found from $\mathrm{Eq}$ (46) $N^{-1} \operatorname{Tr} \mathbf{O}=T$ artanha $a_{0}$ The result for the averages is then

$$
\begin{gathered}
\left\langle\left|\left(\mathbf{r}_{-+}\right)_{n n}\right|^{2}\right\rangle=T^{2}\left|\operatorname{artanh} a_{0}\right|^{2}, \\
\left\langle\left|\left(\mathbf{r}_{-+}\right)_{m n}\right|^{2}\right\rangle=N^{-1}\left\langle R_{-}\right\rangle, \quad m \neq n
\end{gathered}
$$

The incident plane wave is reconstructed with an intensity $\propto T^{2}$, in agreement with Refs $[11,12]$ In the coherent regime, off-diagonal $(m \neq n)$ and diagonal $(m=n)$ reflection probabilities differ by a large factor of order $N T$

In the incoherent regime, the matrices $\mathbf{V}_{+}$and $\mathbf{V}_{-}$are independent Integration over $\mathcal{U}(N)$ results in integrals of the form $\int d \mathbf{V} V_{n k} V_{n l}^{*}=N^{-1} \delta_{l k}$ Then the off-diagonal and $\mathrm{d}_{1-}$ agonal reffection probabilities are both given by

$$
\left\langle\left|\left(\mathbf{r}_{-+}\right)_{m n}\right|^{2}\right\rangle=N^{-1}\left\langle R_{-}\right\rangle
$$

so there is no peak in the reflected intensity at the angle of incidence This holds for every $N$ and $L$

For both the incoherent and the coherent regime we find for the reflection without frequency shift $\left(\omega_{+} \rightarrow \omega_{+}\right)$the probab1lity

$$
\left\langle\left|\left(\mathbf{r}_{++}\right)_{m n}\right|^{2}\right\rangle=\frac{1+\delta_{m n}}{1+N}\left\langle R_{+}\right\rangle
$$

Here we see a much smaller backscattering peak, where the diagonal reflection probability is only twice as large as the off-diagonal reflection probability [26] This factor is independent of the phase conjugating mirror, and exists entirely because of time-reversal symmetry [27]

\section{Numerical simulations}

To test the analytical piedictions of random-matrix theory we have carried out numerical simulations The Helmholtz equation,

$$
\left(-\nabla^{2}-\varepsilon \omega_{ \pm}^{2} / c^{2}\right) \mathcal{E}=0
$$

1s discretized on a square lattice (lattıce constant $d$, length $L$, width $W$ ) Disorder is introduced by letting the relative d1electric constant $\varepsilon$ fluctuate from site to site between $1 \pm \delta \varepsilon$ Using the method of recursive Green functions [23] we compute the scattering matrix $S_{\text {disorder }}(\omega)$ of the disordered medium at frequencies $\omega_{+}$and $\omega_{-}$The reflection matrix $\mathbf{r}_{\mathrm{PCM}}$ of the phase-conjugating mirror is calculated by discretizing Eq (22) From $\mathbf{S}_{\mathrm{d} \text { isorder }}\left(\omega_{ \pm}\right)$and $\mathbf{r}_{\mathrm{PCM}}$ we obtain the reflection matrix $\mathbf{r}$ of the entire system, and hence the reflectances (4 10)

We took $W=51 d, \delta \varepsilon=05, \alpha=\pi / 4$, and varied $\delta$ and $L$ For the coherent case we took $\omega_{+}=\omega_{-}=1252 \mathrm{c} / d$, and for the incoherent case $\omega_{+}=1252 c / d, \omega_{-}=1166 c / d$ These parameters correspond to $N_{+}=22, l_{+}=155 d$ at frequency $\omega_{+}$The mean free path is determined using $\mathrm{Eq}$ (310), which holds up to small corrections of order $N^{-1}$ In the 1ncoherent case we have $N_{-}=20, l_{-}=201 d$ This leads to $\Delta \omega=0043 c / d$ and a dwell time for $L / l \simeq 3$ of 


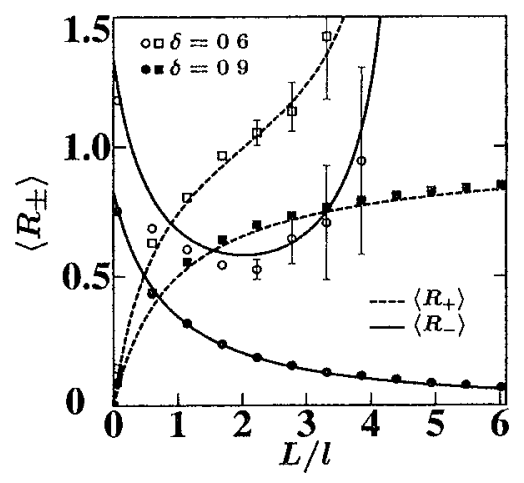

FIG 7 Average reflectances $\left\langle R_{ \pm}\right\rangle$as a function of $L / l$ for $\alpha=\pi / 4$ and $\delta=06,09$, in the incoherent regime The curves are the analytical results for $N \gg 1$, computed from Eq (3 12) Data points are results from numerical simulations (Statistical error bars are shown when they are larger than the size of the marker)

$\tau_{\text {dwell }} \simeq L / c l \approx 150 d / c$ Hence we have $\tau_{\text {dwell }} \Delta \omega \simeq 65$, which should be well in the incoherent regime For comparison with random-matrix theory, we take the large- $N$ limit and use the value $l_{+}$for $l$

The numerical results are shown in Figs 6 (coherent regime) and 7 (incoherent regime), for $\delta=06$ and 09 As we can see, the agreement with the analytical theory is quite satisfactory The rapid rise of $\left\langle R_{ \pm}\right\rangle$in the incoherent regime for the smallest $\delta$ is accompanied by large statistical fluctuations, which make an accurate comparison more difficult Still, the striking differences between the coherent and incoherent regimes predicted by the random-matrix theory are confirmed by the simulations

We have also studied the backscattering peak for planewave illumination We considered a square sample ( $W=L=251 d$ ) with $\alpha=\pi / 4, \delta=09$ We calculated the reflection probabilities $\left|\left(r_{-+}\right)_{m n}\right|^{2}$ for normal incidence $(n=1)$ in both the coherent and the incoherent regimes The numerical results for a single realization of the disorder are shown in Fig 8 The arrow denotes the analytical ensemble average (425) of the backscattering peak in the large- $N$ limit, which is consistent with the numerical data Notice the absence of a backscattering peak in the incoherent tegime

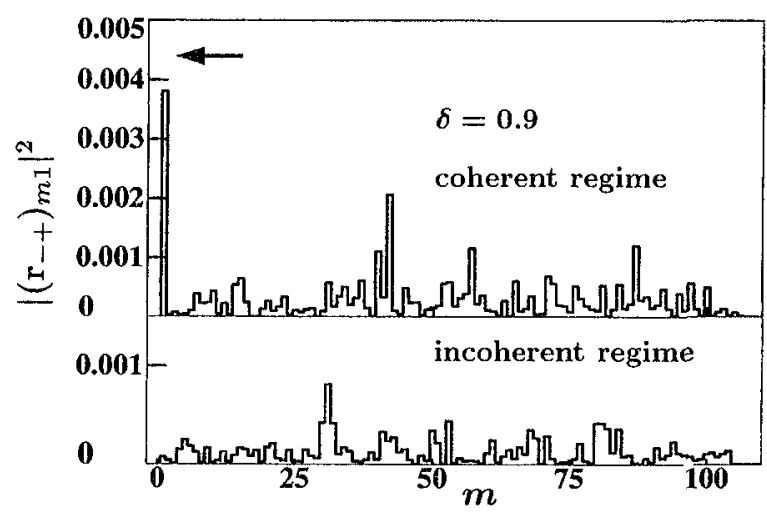

FIG 8 Histograms for the modal distrubution $\left|\left(\mathbf{r}_{-+}\right)_{m 1}\right|^{2}$ of the reflection probability with frequency shift for normal incidence The results ase for a single realization of the disorder at $W=L=251 d(L / l=162), \alpha=\pi / 4$ and $\delta=09$ The arrow ind cates the theoretical value $\left\langle\left|\left(\mathbf{r}_{-+}\right)_{1}\right|^{2}\right\rangle$ trom Eq (425), representing the ensemble average in the large- $N$ limit

\section{COMPARISON WITH ANDREEV REFLECTION}

We have studied the reflection of light by a disordered dielectric medium in front of a phase-conjugating mirror This problem has an electronic andlog $[17,18]$ The electronic disordered system consists of a metal, in which electron or hole excitations are scattered elastically by randomly placed impurities Retroreflection at the phase-conjugating mirror is analogous to Andreev reflection at the interface with a superconductor The Fermı energy $E_{F}$ plays the role of the pump frequency $\omega_{0}$, while the excitation energy $E$ corresponds to the frequency shift $\Delta \omega$ In spite of these sim1larities, the optical effects tound in this paper have no electronic analog It is instructive to see where the analogy breaks down

To this end we compare the wave equation (22) with the Bogoluubov-de Gennes equation [28]

$$
\left(\begin{array}{cc}
H & \Delta \\
\Delta^{*} & -H
\end{array}\right)\left(\begin{array}{l}
u \\
v
\end{array}\right)=E\left(\begin{array}{l}
u \\
v
\end{array}\right) \text {, }
$$

which determines the electron and hole wave functions $u$ and $v$ The Hamiltonian

$$
H=-\frac{\hbar^{2}}{2 m} \nabla^{2}+V-E_{F}
$$

contains the electiostatic potential $V(\mathbf{r})$, which plays the role of the dielectric constant [More precisely, $k_{0}^{2}(\varepsilon-1)$ corresponds to $\left.-\left(2 m / \hbar^{2}\right) V\right]$ The role of the nonlinear susceptıbility is played here by the pair potential $\Delta(\mathbf{r})$, which is only nonzero in the superconductor, where 1 equals $\Delta_{0} e^{i \psi}$ Comparing Eqs (51) and (52) for the electronic case with the optical equations (2 2) and (24) one notices many sim1larities, and some differences which amount to a redefinition of quantities There 1s, however, one essential difference the matrix operator in Eq (5 1) is Hermitian, whlle that in Eq (2) 1s not, because of an extra minus sign in one of the off-diagonal elements This minus sign is the origin of the difference between Andreev reflection and optical phase conjugation

In the optical case a disordered medium becomes transparent $\left(R_{-}=1\right)[9,10]$ for unit reflectance at the phaseconjugating mirror $(a=1)$ This does not happen in the electronic case, where $R_{-}$is reduced by disorder even for ideal Andreev reflection The 1effection matrix of the normalmetal-superconductor (NS) interface, obtaned from $\mathrm{Eq}$ (5 1) for $V \equiv 0, E \ll \Delta_{0} \ll E_{F}$, is given by [1]

$$
\mathbf{r}_{\mathrm{NS}}=\left(\begin{array}{cc}
0 & -\imath e^{-\imath \psi} \\
-\imath e^{\imath \psi} & 0
\end{array}\right)
$$

Comparison with Eq (4 6) for $\mathbf{r}_{\mathrm{PCM}}$ shows that Andreev reflection is independent of the angle of incidence, the matrix a in Eq (4 6) is replaced by the unit matrix in Eq (5 3) This is a substantial simplification of the electronic problem, compared with the optical analog The matrix $\mathbf{r}_{\mathrm{NS}}$ is unitary, in contrast to $\mathbf{r}_{\mathrm{PCM}}$, so that the appearance of gain or loss at the phase-conjugating mirror has no electionic counterpart The 
reflectance $R_{-}=1-R_{+}$is a monotonically decreasing function of $L / l$ in the electronic case [8], both in the coherent regime,

$$
R_{-}=(2+4 L / \pi l)^{-1} \quad \text { if } E \ll \hbar / \tau_{\text {dwell }} \text { and } L \geqslant l,
$$

and in the incoherent regime,

$$
R_{-}=(1+4 L / \pi l)^{-1} \text { if } E \gg \hbar / \tau_{\text {dwell }} .
$$

The result (5.5) is what one obtains from Eq. (3.12) for the case $A=1$ of unit reflectance at the interface. [The transmittance $T=(1+2 L / \pi l)^{-1}$ of the disordered medium is the same for electrons and photons.] The result (5.4), however, is not what one would expect from the optical analog. Indeed, Eq. (4.17) with $a_{0}=1$ would give $R_{-}=1$ for all $L$ in the case of unit reflectance at the phase-conjugating mirror. The reason that the analogy with Andreev reflection breaks down is the difference of a minus sign in the wave equations (2.2) and (5.1), which reappears in the reflection matrices (4.6) and (5.3) for phase conjugation, and ultimately in the reflectances in the coherent regime:

$$
\begin{gathered}
R_{-}=\frac{1}{N} \operatorname{Tr}\left(\frac{\mathbf{t t}^{\dagger}}{1+\mathbf{r r}^{\dagger}}\right)^{2} \neq 1 \text { for electrons, } \\
R_{-}=\frac{1}{N} \operatorname{Tr}\left(\frac{\mathbf{t t}^{\dagger}}{1-\mathbf{r r}^{\dagger}}\right)^{2}=1 \text { for photons if } \mathbf{a}=1 .
\end{gathered}
$$

Here $\mathbf{t}$ and $\mathbf{r}$ are the transmission and reflection matrices of the disordered medium, which satisfy $\mathbf{t t}^{\dagger}+\mathbf{r} \mathbf{r}^{\dagger}=1$.

In conclusion, we have shown that the presence of a phase-conjugating mirror behind a random medium drastically changes the total reflected intensity, even when the medium is so disordered that wave-front reconstruction is ineffective. On increasing the frequency difference $\Delta \omega$ between the incident radiation and the pump beams, a minimum in the disorder dependence of the reflected intensity appears. In a certain parameter range, the disordered medium reflects less radiation on reducing $\Delta \omega$. Experimental observation of this "darkening" would be a striking demonstration of phaseshift cancellations in a random medium.

\section{ACKNOWLEDGMENTS}

This work was supported by the "Stichting voor Fundamenteel Onderzoek der Materie" (FOM) and by the "Nederlandse organisatie voor Wetenschappelijk Onderzoek" (NWO).

\section{APPENDIX: CALCULATION OF THE REFLECTANCES IN THE COHERENT REGIME}

In Sec. IV we computed the average reflectances $\left\langle R_{ \pm}\right\rangle$for the incoherent regime. For the coherent regime we presented only a derivation for scalar reflection matrix a. This appendix contains the calculation of $\left\langle R_{ \pm}\right\rangle$for arbitrary (diagonal) matrix a. Our calculation is based on the diagrammatic method for integration over the unitary group of Refs. $[29,24]$. Integrals over the unitary group are needed for the computation of $\left\langle R_{ \pm}\right\rangle$because of the polar decomposition
(4.11) of the transmission and reflection matrices. We find it convenient to use a slightly modified version of the diagrammatic technique, in which we apply the diagrammatic rules without making explicit use of the polar decomposition. We first outline the calculation of $\left\langle R_{ \pm}\right\rangle$in which the diagrammatic method is used for the integration of the matrices $\mathbf{U}$ and $\mathbf{V}$ in Eq. (4.11), and then discuss the modification of the diagrammatic method.

We start the calculation of $\left\langle R_{ \pm}\right\rangle$with the elimination of the reflection matrix $\mathbf{r}_{11}\left(\omega_{0}\right)$ and the transmission matrices $\mathbf{t}_{12}\left(\omega_{0}\right)$ and $\mathbf{t}_{21}\left(\omega_{0}\right)$ from the reflectances $R_{+}$and $R_{-}$[cf. Eqs. (4.9) and (4.10)], in favor of the matrix $\mathbf{r}=\mathbf{r}_{22}\left(\omega_{0}\right)$. The result is

$$
\begin{aligned}
\left\langle R_{+}\right\rangle= & \frac{1}{N} \operatorname{Tr}\left[\mathbf{s}^{\prime}(\mathbf{a}, \mathbf{a})-\mathbf{s}(1, \mathbf{a})-\mathbf{s}(\mathbf{a}, 1)+\mathbf{s}^{\prime}(1,1)\right. \\
& \left.+\mathbf{h}(1)+\mathbf{h}(1)^{*}\right], \\
\left\langle R_{-}\right\rangle= & \frac{1}{N} \operatorname{Tr}\left\{\mathbf{s}(\mathbf{a}, \mathbf{a})-\mathbf{s}^{\prime}(1, \mathbf{a})-\mathbf{s}^{\prime}(\mathbf{a}, 1)+\mathbf{s}(1,1)\right. \\
& \left.+\mathbf{a}^{-1} \mathbf{a}^{-1 *}\left[1-\mathbf{h}(1)-\mathbf{h}(1)^{*}\right]\right\},
\end{aligned}
$$

where we defined

$$
\begin{gathered}
\mathbf{s}^{\prime}(\mathbf{x}, \mathbf{y})=\left\langle\mathbf{x}\left(1-\mathbf{r a r}^{*} \mathbf{a}\right)^{-1} \mathbf{r y} \mathbf{y}^{*} \mathbf{r}^{*}\left(1-\mathbf{a}^{*} \mathbf{r} \mathbf{a}^{*} \mathbf{r}^{*}\right)^{-1} \mathbf{x}^{*}\right\rangle, \\
\mathbf{s}(\mathbf{x}, \mathbf{y})=\left\langle\mathbf{x}\left(1-\mathbf{r a r}^{*} \mathbf{a}\right)^{-1} \mathbf{a}^{-1} \mathbf{y y}^{*} \mathbf{a}^{-1 *}\left(1-\mathbf{a}^{*} \mathbf{r a}^{*} \mathbf{r}^{*}\right)^{-1} \mathbf{x}^{*}\right\rangle, \\
\mathbf{h}(\mathbf{A} 2 \mathrm{~b})=\left\langle\mathbf{x}\left(1-\mathbf{r a r}^{*} \mathbf{a}\right)^{-1}\right\rangle .
\end{gathered}
$$

To perform the average over $\mathbf{r}$, one may use the polar decomposition [cf. Eq. (4.11)]

$$
\mathbf{r}=i \mathbf{U} \sqrt{1-\mathbf{T}} \mathbf{U}^{\mathrm{T}}
$$

where $\mathbf{U}$ is a unitary matrix and $\mathbf{T}$ is the diagonal matrix containing the $N$ transmission eigenvalues $\tau_{j}$ on the diagonal. The matrix $\mathbf{U}$ is a member of the circular unitary ensemble (CUE), i.e., it is uniformly distributed in the unitary group. The transmission eigenvalues $\tau_{j}$ have density [22]

$$
\begin{gathered}
\rho(\tau)=(2 N / \pi) \operatorname{Im} g(1 / \tau-1-i 0, s), \\
g(\zeta, s)=\operatorname{cotanh}[\zeta-s g(\zeta, s)], \quad s=2 L / \pi l
\end{gathered}
$$

To integrate the matrix $\mathbf{U}$ over the unitary group, the matrices $\mathbf{S}, \mathbf{s}^{\prime}$, and $\mathbf{h}$ are first expanded as a power series in $\mathbf{U}$. The integration of $\mathbf{U}$ is then done using the general expression for the average of a polynomial function of $\mathbf{U}[30]$,

$$
\begin{aligned}
& \left\langle\mathbf{U}_{a_{1} b_{1}} \cdots \mathbf{U}_{a_{m} b_{m}} \mathbf{U}_{\alpha_{1} \beta_{1}}^{*} \cdots \mathbf{U}_{\alpha_{n} \beta_{n}}^{*}\right\rangle \\
& \quad=\delta_{m, n} \sum_{P, P^{\prime}} V_{c_{1}, \ldots, c_{k}} \prod_{j=1}^{n} \delta_{a_{j}, \alpha_{P(j)}} \delta_{b_{j}, \beta_{P^{\prime}(j)}} .
\end{aligned}
$$

Here the summation is over all permutations $P$ and $P^{\prime}$ of the numbers $1, \ldots, n$. The numbers $c_{1}, \ldots, c_{k}$ denote the cycle structure of the permutation $P^{-1} P^{\prime}$. (The permutation $P^{-1} P^{\prime}$ can be uniquely written as a product of disjoint cy- 
clic permutations of lengths $c_{1}, \ldots, c_{k}$, with $n=\sum_{J=1}^{k} c_{k}$.) To compute $\left\langle R_{ \pm}\right\rangle$in the limit of large $N$, it is sufficient to know the coefficients $V_{c_{1}}, \quad, c_{k}$ to leading order in $N$. These are given in Refs. [29,24], together with a diagrammatic method which enables one to restrict the summation over $P$ and $P^{\prime}$ to those permutations $P$ and $P^{\prime}$ of which the contribution to $\left\langle R_{ \pm}\right\rangle$is of maximal order in $N$.

Although the computation of $\left\langle R_{ \pm}\right\rangle$is straightforward now, the actual calculation is rather cumbersome. We find it convenient to modify the approach of Refs. $[29,24]$ so that it can be applied directly to the average over the matrix $\mathbf{r}$, without making explicit use of the polar decomposition (A3). This is possible because the general structure (A5) already follows from the invariance of the distribution of $\mathbf{U}$ under transformations

$$
\mathbf{U} \rightarrow \mathbf{V U V} \mathbf{V}^{\prime}
$$

where $\mathbf{V}$ is an arbitrary unitary matrix. The fact that $\mathbf{U}$ itself is unitary is necessary to compute the value of the coefficients $V_{c_{1}}, \quad, c_{k}$, but it is not relevant for the general structure (A5). Since the matrix $\mathbf{r}$ is both unitary and symmetric, its distribution is invariant under transformations

$$
\mathbf{r} \rightarrow \mathbf{V r V}^{T}
$$

that respect the symmetry of $\mathbf{r}$. The same group of transformations leaves invariant the circular orthogonal ensemble $(\mathrm{COE})$, consistıng of uniformly distributed unitary and symmetric matrices. A diagrammatic technique for averages over the COE is presented in Ref. [24]. As before, the general structure of the average of a polynomial of a matrix from the COE is entirely determined by the invariance under the transformations (A7), and therefore applies to the reflection matrix $\mathbf{r}$ as well. It reads [24,31]

$$
\begin{array}{r}
\left\langle\mathbf{r}_{a_{1} a_{2}} \cdots \mathbf{r}_{a_{2 n-1} a_{2 n}} \mathbf{r}_{\alpha_{1} \alpha_{2}}^{*} \cdots \mathbf{r}_{\alpha_{2 m-1} \alpha_{2 m}}^{*}\right\rangle \\
=\delta_{n, m} \sum_{P} V_{c_{1}, \quad, c_{k}} \prod_{j=1}^{2 n} \delta_{a_{j}, \alpha_{P(J)}},
\end{array}
$$

where now the summation is over permutations $P$ of the numbers $1, \ldots, 2 n$. We may write $P$ as

$$
P=\left(\prod_{j=1}^{n} \sigma_{j}\right) P_{e} P_{o}\left(\prod_{j=1}^{n} \sigma_{j}^{\prime}\right),
$$

where the permutations $\sigma_{j}$ and $\sigma_{j}$ operate on the numbers $2 j-1$ and $2 j$, and the permutation $P_{e}\left(P_{o}\right)$ permutes even (odd) numbers only. The numbers $c_{1}, \ldots, c_{k}$ in Eq. (A8) are the cycle structure of the permutation $P_{e}^{-1} P_{o}$. The specific values of the coefficients $V_{c_{1},}, c_{k}$ for an average of $\mathbf{r}$ are of course different from those for the COE.

Now that we have identified the formal equivalence of an average over the (nonunitary) symmetric reflection matrix $\mathbf{r}$ and a unitary symmetric matrix from the COE, we can directly apply the diagrammatic rules of Refs. $[29,24]$ to an average over the matrix $\mathbf{r}$, provided we know the coefficients $V_{c_{1}}, \quad, c_{k}$ for the ensemble of reflection matrices $\mathbf{r}$ of a dis-

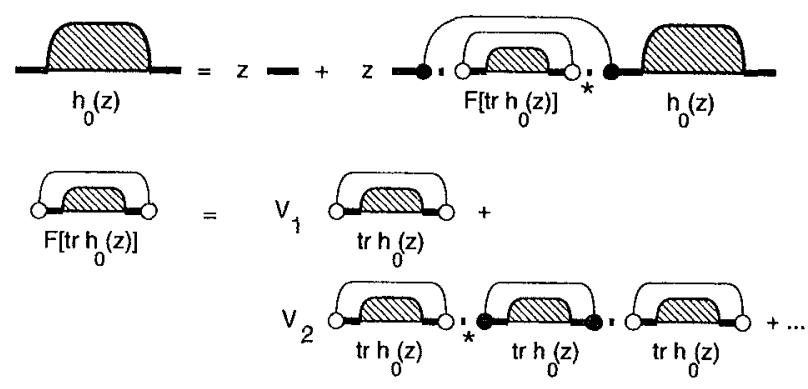

FIG. 9. Diagrams for the calculation of $\mathbf{h}_{0}(z)$.

ordered waveguide. To find these coefficients, we use the fact that they factorize, to leading order in $N$,

$$
V_{c_{1},}, c_{k}=\prod_{j=1}^{k} V_{c_{h}},
$$

just as they do for the COE. This follows directly from the fact that, to leading order in $N$, the average $\left\langle\Pi_{J} \operatorname{Tr}\left(\mathbf{r r}^{\dagger}\right)^{c} J\right\rangle$ factorizes into $\Pi_{j}\left\langle\operatorname{Tr}\left(\mathbf{r r}^{\dagger}\right)^{c^{c}}\right\rangle$ [32]. It remains to find the coefficients $V_{1}$. Hereto we consider the function

$$
\mathbf{h}_{0}(z)=\left\langle\frac{z}{1-\mathbf{r r}^{*} z^{2}}\right\rangle \text {. }
$$

We first compute $\mathbf{h}_{0}(z)$ from the diagrammatic technique, with a priori unknown coefficients $V_{\iota}$. We then compare our result with a calculation of $\operatorname{Tr} \mathbf{h}_{0}(z)$ from the density of transmission eigenvalues (A4). The relevant diagrams for the diagrammatic calculation are shown in Fig. 9 (for a detailed explanation of the diagrammatic notation of Fig. 9, we refer to Ref. [24]). The result is a self-consistency equation for $\mathbf{h}_{0}(z)$ that involves the generating function $F$ of the coefficients $V_{c}$,

$$
\begin{gathered}
\mathbf{h}_{0}(z)=\frac{z \mathbb{H}}{1-z F\left[\operatorname{Tr} \mathbf{h}_{0}(z)\right]}, \\
F(x)=\sum_{j=c}^{\infty} V_{c} x^{2 c-1} .
\end{gathered}
$$

Here 1 is the $N \times N$ unit matrix. Direct computation of $\operatorname{Tr} \mathbf{h}_{0}(z)$ from the density $\rho(\tau)$ of transmission eigenvalues gives

$$
\operatorname{Tr} \mathbf{h}_{0}(z)=\int_{0}^{1} d \tau \frac{\rho(\tau) z}{1-z^{2}(1-\tau)} .
$$

Together, Eqs. (A10)-(A14) determine the coefficients $V_{c_{1}}, \quad, c_{k}$ needed for the diagrammatic evaluation of $\left\langle R_{ \pm}\right\rangle$. In the limit of $L \rightarrow \infty$, the density of transmission eigenvalues tends to $N \delta(\tau)$. Hence $\mathbf{h}_{0}(z)=z /\left(1-z^{2}\right)$ and $F(x)=\left(\sqrt{N^{2}+4 x^{2}}-N\right) / 2 x$. The corresponding coefficients $V_{c}=c^{-1} N^{1-2 c}\left(\begin{array}{c}c-1 \\ 2 c-2\end{array}\right)$ are precisely those of the COE [24]. For finite $L$, the density $\rho(\tau)$ is no longer a $\delta$ function, and hence the coefficients $V_{c}$ deviate from those of the COE.

The fact that we can use the diagrammatic rules directly for the average over $\mathbf{r}$ simplifies the calculation considerably. A central role in the calculation is played by the function 
$\mathbf{h}(\mathbf{x})$ defined in Eq. (A2). The diagrams for the calculation of $\mathbf{h}(\mathbf{x})$ are similar to those of Fig. 9, and the result is a selfconsistency equation for $\mathbf{h}(\mathbf{x})$,

$$
\mathbf{h}(\mathbf{x})=\mathbf{x}(1-\mathbf{a} F[\operatorname{Tr} \mathbf{h}(\mathbf{a})])^{-1} .
$$

Notice the formal equivalence with Eq. (A12). The function $F$ was defined in Eq. (A13). Using the diagrammatic technique for the computation of $s$ and $s^{\prime}$, we find the linear relations

$$
\begin{gathered}
\mathbf{s}(\mathbf{x}, \mathbf{y})=\mathbf{h}(\mathbf{x})\left[\mathbf{a}^{-1} \mathbf{y y}^{*} \mathbf{a}^{-1} *+K \operatorname{Tr} \mathbf{s}^{\prime}(\mathbf{a}, \mathbf{y})\right. \\
+L \operatorname{Tr} \mathbf{s}(\mathbf{a}, \mathbf{y})] \mathbf{h}(\mathbf{x})^{*}, \\
\mathbf{s}^{\prime}(\mathbf{x}, \mathbf{y})=\mathbf{h}(\mathbf{x})\left[K \operatorname{Tr} \mathbf{s}(\mathbf{a}, \mathbf{y})+L \operatorname{Trs}^{\prime}(\mathbf{a}, \mathbf{y})\right] \mathbf{h}(\mathbf{x})^{*},
\end{gathered}
$$

where we have defined

$$
\begin{gathered}
h=\operatorname{Tr} \mathbf{h}(\mathbf{a}), \\
K=\sum_{i, j=1}^{\infty} V_{i+j-1} h^{2 i-2}\left(h^{*}\right)^{2 j-2}=\frac{h F(h)-h^{*} F(h)^{*}}{h^{2}-h^{* 2}}, \\
L=\sum_{i, j=1}^{\infty} V_{i+j} h^{2 i-1}\left(h^{*}\right)^{2 j-1}=\frac{h^{*} F(h)-h F(h)^{*}}{h^{2}-h^{* 2}} .
\end{gathered}
$$

The relevant diagrams leading to Eqs. (A16) and (A17) are shown in Fig. 10. They are similar to those of Ref. [29], where the case of a chaotic cavity was considered, instead of a disordered waveguide. Together, Eqs. (A15)-(A17) form a closed set of equations, from which $\mathbf{s}(\mathbf{x}, \mathbf{y}), \mathbf{s}^{\prime}(\mathbf{x}, \mathbf{y})$, and $\mathbf{h}(\mathbf{x})$ can be calculated. The average reflectances $\left\langle R_{ \pm}\right\rangle$are obtained upon substitution of $\mathbf{s}(\mathbf{x}, \mathbf{y}), \mathbf{s}^{\prime}(\mathbf{x}, \mathbf{y})$, and $\mathbf{h}(\mathbf{x})$ into Eq. (A1). The final result is expressed as a function of $h=\operatorname{Tr} \mathbf{h}(\mathbf{a})$,

$$
\begin{gathered}
N\left\langle R_{+}\right\rangle=\frac{\left(I^{2}+J^{2}\right) K-2 J(1-I L)}{(1-I L)^{2}-(K I)^{2}} \\
+2 \operatorname{Re} \operatorname{Tr}[1-\mathbf{a} F(h)]^{-1}, \quad(\mathrm{~A} 18) \\
N\left\langle R_{-}\right\rangle=\frac{\left(I+L J^{2}\right)(1-I L)+K I J(K I-2)}{(1-I L)^{2}-(K I)^{2}}+J|F(h)|^{2},
\end{gathered}
$$

where we defined
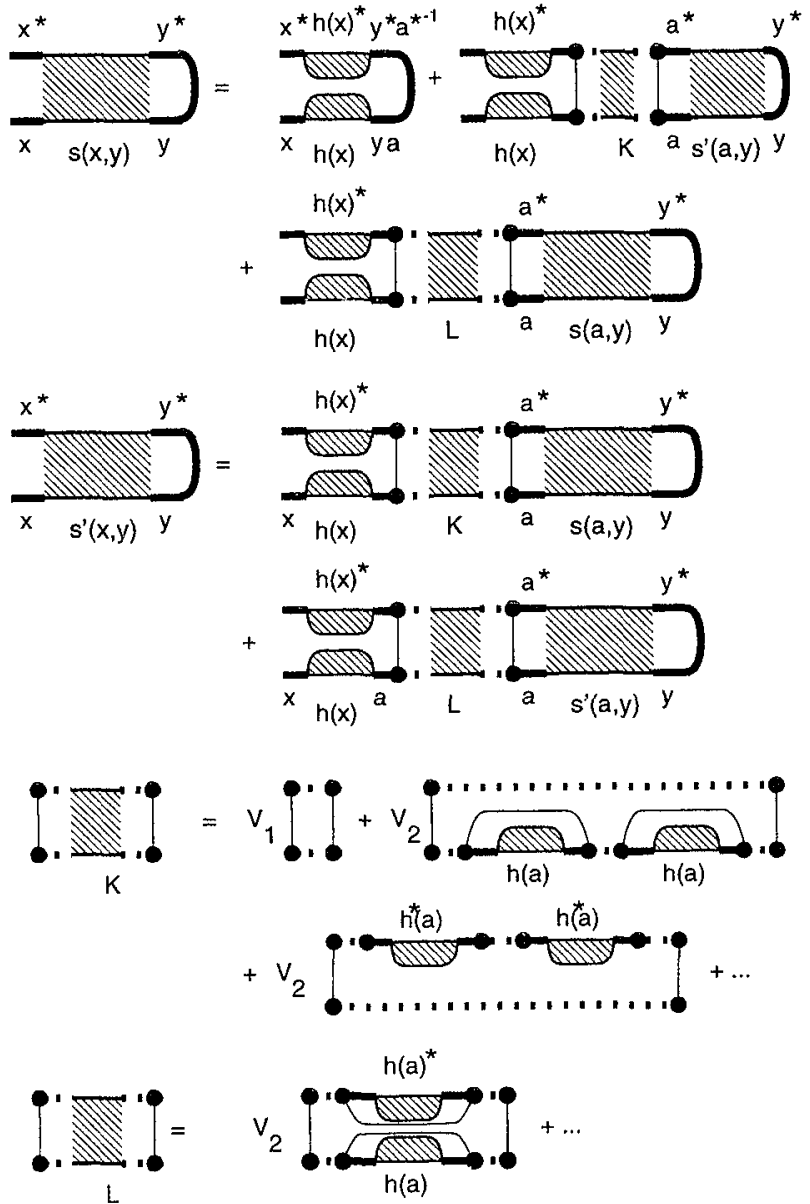

FIG. 10. Diagrams for the calculation of $\mathbf{s}(\mathbf{x}, \mathbf{y})$ and $\mathbf{s}^{\prime}(\mathbf{x}, \mathbf{y})$.

$$
\begin{gathered}
I=\operatorname{Tr} \mathbf{a}[1-\mathbf{a} F(h)]^{-1}\left[1-\mathbf{a}^{*} F(h)^{*}\right]^{-1} \mathbf{a}^{*}, \\
J=\operatorname{Tr}[1-\mathbf{a} F(h)]^{-1}\left[1-\mathbf{a}^{*} F(h)^{*}\right]^{-1} .
\end{gathered}
$$

These expressions simplify in the large- $L / l$ limit, when $\rho(\tau)$ takes the form (4.18). Substitution in Eq. (A14) gives

$$
\begin{gathered}
\operatorname{Tr} \mathbf{h}_{0}(z)=\frac{N z}{1-z^{2}}\left(1-\frac{z \operatorname{artanh} z}{1+s}\right)+O(1+s)^{2}, \\
s=2 L / \pi l
\end{gathered}
$$

and hence allows us to find $F(z)$ from Eq. (A12). Expanding the expressions (A18) for $\left\langle R_{ \pm}\right\rangle$and the self-consistency equation (A15) to lowest order in $(1+s)^{-1}$ we find the results (4.19) and (4.20), with the effective reflectance $a_{0}=z$.
[1] A. F. Andreev, Zh. Eksp. Teor. Fiz. 46, 1823 (1964) [Sov. Phys. JETP 19, 1228 (1964)]; 49, 655 (1965) [22, 455 (1966)].

[2] J. P. Woerdman, Opt. Commun. 2, 212 (1970).

[3] B. I. Stepanov, E. V. Ivakin, and A. S. Rubanov, Dokl. Akad. Nauk USSR 196, 567 (1971) [ Sov. Phys. Dokl. 16, 46 (1971)].

[4] A. A. Abrikosov, Fundamentals of the Theory of Metals
(North-Holland, Amsterdam, 1988).

[5] Optical Phase Conjugation, edited by R. A. Fisher (Academic, New York, 1983).

[6] B. Ya. Zel'dovich, N. F. Pilipetskiı̌, and V. V. Shkunov, Principles of Phase Conjugation (Springer, Berlin, 1985).

[7] D. M. Pepper, Sci. Am. 254, 56 (1986).

[8] C. W. J. Beenakker, in Mesoscopic Quantum Physics, edited 
by E Akkermans, G Montambaux, J -L Pichard, and J ZinnJustin (North-Holland, Amsterdam, 1995)

[9] Yu N Barabanenkov, Yu A Kravtsov, V D Ozrin, and A I Sarchev, in Progress in Optıcs XXIX, edited by E Wolf (North-Holland, Amsterdam, 1991)

[10] R Mittra and T M Habashy, J Opt Soc Am A 1, 1103 (1984)

[11] I McMichael, M D Ewbank, and F Vachss, Opt Commun 119, 13 (1995)

[12] C Gu and P Yeh, Opt Commun 107, 353 (1994)

[13] S Chandrasekhar, Radlative Transfer (Dover, New York, 1960)

[14] A Ishmaru, Wave Propagation and Scattering in Random Media (Academic, New York, 1978)

[15] J C J Paasschens, P W Brouwer, and C W J Beenakker, Europhys Lett 38, 651 (1997)

[16] I Freund, M Rosenbluh, R Berkovits, and M Kaveh, Phys Rev Lett 61, 1214 (1988), M I Mishchenko, J M Dlugach, and E G Yanovitski], J Quant Spectrosc Radiat Transf 47, 401 (1992)

[17] D Lenstra, in Huygens Principle 1690-1990, Theory and Appllcations, edited by $\mathrm{H}$ Blok, $\mathrm{H}$ A Ferweda, and $\mathrm{H} \mathrm{K}$ Kuiken (North-Holland, Amsterdam, 1990)

[18] H van Houten and C W J Beenakker, Physica B 175, 187 (1991)
[19] A Yariv and D M Pepper, Opt Lett 1, 16 (1977)

[20] H F Arnoldus and T F George, J Mod Opt 36, 31 (1989)

[21] M J M de Jong, Phys Rev B 49, 7778 (1994)

[22] Two reviews of the random-matrix theory of phase-coherent scattering are A D Stone, P A Mello, K A Muttalıb, and J -L Pichard, in Mesoscopic Phenomena in Sollds, edited by B L Altshuler, P A Lee, and R A Webb (North-Holland, Amsterdam, 1991), C W J Beenakker, Rev Mod Phys 69, 731 (1997)

[23] H U Baranger, D P DiVincenzo, R A Jalabert, and A D Stone, Phys Rev B 44, 10637 (1991)

[24] P W Brouwer and C W J Beenakker, J Math Phys (N Y) 37, 4904 (1996)

[25] C W J Beenakker, J A Melsen, and P W Brouwer, Phys Rev B 51, 13883 (1995)

[26] P A Mello, E Akkermans, and B Shapıro, Phys Rev Lett 61, 459 (1988)

[27] G Bergmann, Phys Rep 107, 1 (1984)

[28] P G de Gennes, Superconductuvty of Metals and Alloys (Benjamın, New York, 1966)

[29] N Argaman and A Zee, Phys Rev B 54, 7406 (1996)

[30] S Samuel, J Math Phys (N Y ) 21, 2695 (1980)

[31] P A Mello and T H Seligman, Nucl Phys A 344, 489 (1980)

[32] P A Mello and A D Stone, Phys Rev B 44, 3559 (1991) 